Philadelphia College of Osteopathic Medicine DigitalCommons@PCOM

PCOM Scholarly Papers

2014

\title{
Safety and Efficacy of Ceftaroline Fosamil in the Management of Community-Acquired Bacterial Pneumonia
}

Heather F. DeBellis

Kimberly L. Barefield

Philadelphia College of Osteopathic Medicine, kimberlba@pcom.edu

Follow this and additional works at: https://digitalcommons.pcom.edu/scholarly_papers

Part of the Medicine and Health Sciences Commons

\section{Recommended Citation}

DeBellis, Heather F. and Barefield, Kimberly L., "Safety and Efficacy of Ceftaroline Fosamil in the Management of CommunityAcquired Bacterial Pneumonia" (2014). PCOM Scholarly Papers. 1913.

https://digitalcommons.pcom.edu/scholarly_papers/1913 


\title{
Clinical Medicine Reviews in Therapeutics
}

\section{Safety and Efficacy of Ceftaroline Fosamil in the Management of Community- Acquired Bacterial Pneumonia}

\author{
Heather F. DeBellis and Kimberly L. Tackett \\ South University School of Pharmacy, Savannah, GA, USA.
}

\begin{abstract}
Ceftaroline fosamil is a new fifth-generation cephalosporin indicated for the treatment of community-acquired bacterial pneumonia (CABP). It possesses antimicrobial effects against both Gram-positive and Gram-negative bacteria, including methicillin-resistant Staphylococcus aureus (MRSA), but not against anaerobes. Organisms covered by this novel agent that are commonly associated with CABP are Streptococcus pneumoniae, Staphylococcus aureus, Haemophilus influenzae, Moraxella catarrbalis, and Klebsiella pneumoniae; however, ceftaroline fosamil lacks antimicrobial activity against Pseudomonas and Acinetobacter species. FOCUS 1 and FOCUS 2 clinical trials evaluated the use of ceftaroline fosamil in the treatment of CABP as compared to ceftriaxone. These non-inferiority trials provided evidence that ceftaroline fosamil is as effective and safe as ceftriaxone in the treatment of CABP. As its role in the treatment has not been well established, ceftaroline fosamil should be reserved for patients at high risk for multidrug-resistant organisms (MDROs). This review summarizes ceftaroline fosamil's pharmacokinetic and pharmacodynamic profile, clinical efficacy and safety, and place in therapy for the treatment and management of CABP.
\end{abstract}

KEYWORDS: ceftaroline fosamil, community-acquired bacterial pneumonia, cephalosporins, MRSA, S. pneumoniae

CITATION: DeBellis and Tackett. Safety and Efficacy of Ceftaroline Fosamil in the Management of Community-Acquired Bacterial Pneumonia. Clinical Medicine Reviews in Therapeutics 2014:6 1-6 doi: 10.4137/CMRT.S9346.

RECEIVED: October 7, 2013. RESUBMITTED: January 12, 2014. ACCEPTED FOR PUBLICATION: January $13,2014$.

ACADEMIC EDITOR: Garry Walsh, Editor in Chief

TYPE: Review

FUNDING: Author(s) disclose no funding sources.

COMPETING INTERESTS: Author(s) disclose no potential conflicts of interest.

COPYRIGHT: () Libertas Academica Ltd.

CORRESPONDENCE: hdebellis@southuniversity.edu; ktackett@southuniversity.edu

\section{Introduction}

Pneumonia cases in 2006 collectively contributed to 1.2 million hospitalizations, which eventually led to over 55,000 patient deaths in the same year from the disease. When combined with influenza, pneumonia is the eighth leading cause of death in the US. Approximately 5.6 million US patients are infected with community-acquired bacterial pneumonia (CABP) annually, contributing to costs exceeding $\$ 12$ billion yearly. ${ }^{1}$

$\mathrm{CABP}$ is defined as an alveolar infection that develops in the outpatient setting or within 48 hours of hospital admission and is primarily caused by Streptococcus pneumoniae; however, other culprits include Haemophilus influenzae, Moraxella catarrbalis, Mycoplasma pneumoniae, and Chlamydophila pneumoniae. ${ }^{1}$ In contrast, healthcare-associated pneumonia (HCAP) cases are mainly infected by Legionella pneumophila, Staphylococcus aureus, or other bacteria. ${ }^{2}$ The specific pathogenic organisms vary by patient populations and may be influenced by the disease states of the patient. This can be additionally challenging for practitioners to identify and appropriately treat since certain risk factors and comorbidities (eg, alcoholism, COPD, smoking, aspiration, and HIV infection) can make patients more susceptible to uncommon causative organisms. ${ }^{2}$

Physician adherence to the Infectious Diseases Society of America/American Thoracic Society (IDSA/ATS) treatment guidelines for CABP is paramount because it improves patient outcomes. According to the current IDSA/ATS treatment guidelines for $\mathrm{CABP}$, there are different empiric treatment options for CABP in the outpatient setting. Nonhospitalized patients who are otherwise healthy should receive monotherapy with a macrolide or doxycycline. In those with comorbid conditions such as the presence of chronic disease, 
recent antimicrobial use, or risk factors for drug-resistant S. pneumoniae, a respiratory fluoroquinolone (levofloxacin, moxifloxacin, or gemifloxacin) or a $\beta$-lactam plus a macrolide is recommended. If a patient is admitted to the hospital, a respiratory fluoroquinolone or a macrolide plus a $\beta$-lactam is suggested. Additional antibiotic selection can be tailored to the suspected bacteria, patient risk factors, and environmental risk factors (eg, admission to the intensive care unit). Practitioners should encourage smoking cessation and promote pneumococcal and influenza vaccinations in order to aid in the prevention of CABP. ${ }^{2,3}$

In the last several years, owing to concerns regarding multidrug-resistant organisms (MDROs), a new antibiotic has been developed and approved for use in the treatment of CABP. Ceftaroline fosamil (Teflaro ${ }^{\circledR}$ ) was approved by the US Food and Drug Administration (FDA) in October 2010. Ceftaroline fosamil is a new extended-spectrum cephalosporin, $\beta$-lactam antibiotics, with activity against methicillin-resistant Staphylococcus aureus (MRSA) and penicillin-resistant Streptococcus pneumoniae. ${ }^{3-5}$ It also has Gram-negative coverage against Escherichia coli, Proteus mirabilis, $H$. influenzae, $M$. catarrbalis, Neisseria meningitidis, as well as wild-type Enterobacteriaceae, but has no activity against anaerobes. This review summarizes ceftaroline fosamil's pharmacokinetic and pharmacodynamic profile, clinical efficacy and safety, and place in therapy for the treatment and management of CABP.

\section{Mechanism of Action}

Ceftaroline fosamil is classified as a fifth-generation cephalosporin. It is a prodrug that was developed by modifying the structure of a fourth-generation cephalosporin, cefozopran. ${ }^{5-7}$ The antibacterial activity of ceftaroline occurs through binding to penicillin-binding proteins (PBPs) and interrupting cell wall synthesis, similar to other $\beta$-lactam antibiotics. ${ }^{7-9}$ It binds to PBPs 1-4 with an especially high affinity for PBP2a, which is associated with methicillin resistance. An oxine ring provides ceftaroline with activity against $\beta$-lactamase producing microorganisms and a 1,2,4-thiadiazole ring gives it Gram-negative activity. Ceftaroline provides improved activity against $S$. aureus and $S$. pneumoniae as a result of the $3^{\prime}$ side chain mediating improved binding to PBPs that exhibit decreasing binding affinity for standard $\beta$-lactams. ${ }^{10}$

\section{Antimicrobial Activity}

Ceftaroline exhibits both bactericidal and time-dependent killing activity. ${ }^{4}$ Antimicrobial effects are a result of binding and irreversible inactivation of essential PBPs, leading to the inhibition of bacterial cell-wall biosynthesis, lysis, and death. It has a broad spectrum of activity including both Grampositive and Gram-negative bacteria as well as MRSA. ${ }^{8}$ The spectrum of activity against Gram-negative bacilli is similar to that seen with ceftriaxone. ${ }^{11}$ Susceptible bacterial isolates from patients enrolled in the FOCUS 1 and 2 studies along with the minimum inhibitory concentration (MIC) ranges are listed in Table $1 .^{12}$ Ceftaroline has a bacterial spectrum of activity against organisms that are commonly associated with CABP including $S$. pneumoniae, S. aureus, $H$. influenzae, M. catarrhalis, and Klebsiella pneumoniae, but does not possess activity against atypical pathogens. ${ }^{12}$ Resistance to CABP pathogens has been an increasing concern, promoting the need for the development of newer antibiotics for the treatment of CABP. A 12-year analysis of pneumococcal resistance rates has shown an increase in resistance to commonly used $\beta$-lactam agents. ${ }^{4,10,11,13}$

The Gram-positive anaerobic activity of ceftaroline is similar to that seen with amoxicillin-clavulanate. ${ }^{9}$ It has limited activity against the Bacteroides fragilis group, but does have coverage against $\beta$-lactamase negative strains. In addition to activity against MRSA, ceftaroline exhibits in vitro activity against vancomycin-intermediate and vancomycin-resistant S. aureus. ${ }^{8}$ Ceftaroline has limited activity against Enterococcus faecalis, Enterococcus faecium, and Corynebacterium jeikeium.

Ceftaroline is still susceptible to extended spectrum $\beta$-lactamases, cephalosporinases, and carbapenemases. ${ }^{14}$ There are current ongoing studies on the drug in combination with the $\beta$-lactamase inhibitor, avibactam, in order to expand its antimicrobial coverage. ${ }^{4,14}$ The addition of avibactam restores ceftaroline's activity against Enterobacteriaceae strains that are resistant to other broad spectrum $\beta$-lactam antibiotics.

\section{Pharmacokinetics}

Following intravenous (IV) administration, the conversion of the prodrug ceftaroline fosamil to active form ceftaroline is dependent upon metabolism by phosphatase enzymes in the plasma. ${ }^{5-7} \mathrm{~A}$ small portion of the active drug then undergoes metabolism via hydrolysis to the $\beta$-lactam ring to form an inactive metabolite, ceftaroline-M-1. ${ }^{9}$ Ceftaroline and its metabolites are primarily eliminated by the kidneys with a renal clearance of 95.6 and $86.7 \mathrm{~mL} /$ minute for single and multiple doses, respectively. ${ }^{5,9,15}$ Approximately $88 \%$ of the drug was found in the urine and $6 \%$ in the feces 48 hours following the administration of a $600 \mathrm{mg}$ dose,. ${ }^{5,8}$ In vitro studies indicate that ceftaroline is not metabolized by cytochrome $\mathrm{P}-450$ isoenzymes, resulting in less drug interactions and no dosing adjustments for impaired hepatic function. ${ }^{4}$

Table 1. Antimicrobial spectrum of activity. ${ }^{12,18-21}$

\begin{tabular}{|ll|}
\hline ORGANISM & MIC RANGE (MG/L) \\
\hline S. pneumoniae & $\leq 0.015-0.12$ \\
\hline S. pneumoniae (multidrug resistant) & $\leq 0.015-0.12$ \\
\hline S. aureus & $0.12-0.5$ \\
\hline H. influenzae & $\leq 0.015-0.5$ \\
\hline Haemophilus parainfluenzae & $\leq 0.015-1$ \\
\hline E. coli & $0.03->16$ \\
\hline K. pneumoniae & $0.06-0.5$ \\
\hline
\end{tabular}


Single and multiple dose studies show that ceftaroline displays linear pharmacokinetics with proportional increases in the maximum concentration ( $\mathrm{Cmax}$ ) and area under the plasma drug concentration-time curve (AUC) with increases

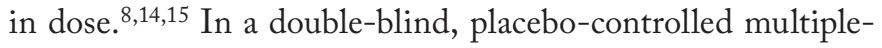
ascending dose study, the AUC and Cmax of ceftaroline increased proportionately with dose following the IV administration of 300 or $600 \mathrm{mg}$ every 12 hours or $800 \mathrm{mg}$ every 24 hours for multiple days. ${ }^{16,17}$ There has been shown to be no ceftaroline accumulation following the administration of multiple doses at either 12 or 24 hours for up to 14 days. ${ }^{7}$ Plasma protein binding is approximately $20 \%$ and decreases with increasing concentrations. ${ }^{6,7,9}$ The median steady-state volume of distribution following a single $600 \mathrm{mg}$ dose is $20.3 \mathrm{~L}$ with a range of 18.3-21.6 L, which is similar to extracellular fluid volume, indicating distribution into total body fluids. Body weight is a predictor of volume of distribution while creatinine clearance $(\mathrm{CrCl})$ can be used to predict drug elimination. ${ }^{8}$

The elimination half-life of ceftaroline is estimated to be 2.6 hours in patients with normal renal function; the halflife of the inactive metabolite is 4.5 hours. ${ }^{5-7,9}$ The AUC and half-life increase by an estimated 25 and 14\%, respectively, in patients with mild renal impairment (estimated $\mathrm{CrCl}$ 50-80 $\mathrm{mL} / \mathrm{minute})$, and the AUC increases by up to $50 \%$ in patients with moderate to severe renal impairment (estimated $\mathrm{CrCl}$ 30-50 mL/minute). ${ }^{5,6}$ Therefore, dosage adjustment is recommended in patients with moderate to severe renal impairment and with end-stage renal disease (ESRD) receiving hemodialysis (estimated $\mathrm{CrCl} 15-50 \mathrm{~mL} /$ minute). The manufacturer recommends that ceftaroline doses be scheduled for administration following dialysis as the drug is removed during hemodialysis. ${ }^{5}$ In vitro studies indicate that ceftaroline is not metabolized by cytochrome P-450 isoenzymes, resulting in less drug interactions and no adjustments for impaired hepatic function. ${ }^{4}$

Elderly patients may require a dosage adjustment for ceftaroline fosamil because of age-related changes in renal function. ${ }^{5}$ The mean AUC and half-life were shown to be increased by 33 and $41 \%$, respectively, in healthy elderly patients $(\geq 65$ years of age) compared to healthy young adults (18-45 years of age) following a single $600 \mathrm{mg}$ dose. ${ }^{5}$

There is limited data establishing the safety and efficacy of ceftaroline fosamil in pediatric patients ( $<18$ years of age). ${ }^{5}$ The pharmacokinetics of ceftaroline are similar in adolescents to those observed in healthy adult patients. In patients of 12-17 years of age with normal renal function, the mean plasma clearance and volume of distribution following an $8 \mathrm{mg} / \mathrm{kg}$ dose were similar to those observed in healthy adult subjects after receiving a $600 \mathrm{mg}$ dose. However, Cmax and AUC were reduced by 10 and 23\%, respectively. ${ }^{5}$

\section{Pharmacodynamics}

Ceftaroline exhibits bactericidal, time-dependent, and concentration-independent killing. ${ }^{6,8}$ The primary predictor of bacteriologic and clinical efficacy is the percentage of time that the free drug remains above the MIC for the pathogen. ${ }^{8}$ The time the free drug remains above the MIC for staphylococci is $30 \%$ of the total administration time for ceftaroline to be bacteriostatic and 40\% for S. pneumoniae and Gram-negative bacilli. ${ }^{4,8,9}$ Ceftaroline demonstrates bactericidal activity when the time the free drug remains above the MIC reaches 50\% in staphylococci and 60\% in Gram-negative bacilli. Neither protein binding nor methicillin and penicillin resistance have been shown to affect this parameter. Ceftaroline demonstrates a limited post-antibiotic effect against most pathogens except $S$. aureus, against which it has been shown to possess a longer effect.

\section{Clinical Efficacy}

The FOCUS 1 and FOCUS 2 clinical trials are randomized, double-blind, and multicenter phase III studies evaluating the safety and efficacy of ceftaroline fosamil when compared to ceftriaxone for the treatment of patients with CABP. Results of the FOCUS 1 and 2 studies are summarized in Tables 2 and 3. Study participants were randomized (1:1) to receive drug therapy for 5-7 days of either ceftaroline fosamil $600 \mathrm{mg}$ every 12 hours or ceftriaxone $1 \mathrm{~g}$ every 24 hours if they were hospitalized with CABP, requiring IV therapy, and having a pneumonia outcomes research team (PORT) risk class score of III or IV. The FOCUS 1 and FOCUS 2 clinical trials were intended as parallel, methodologically similar (with the exclusion of two doses of clarithromycin given on day 1 during FOCUS 1 only) comparative analyses, which did not permit change to oral medications for drug therapy completion. ${ }^{3,18,19}$

The primary endpoint of the FOCUS program was to determine the non-inferiority of ceftaroline fosamil in clinical cure rates when compared with ceftriaxone in the clinically evaluable (CE) and modified intent-to-treat efficacy (MITTE) populations at the test-of-cure (TOC) visit. A clinical cure was defined as an afebrile condition for 24 consecutive hours and conclusion of signs and symptoms of CABP or determination that antibiotic therapy was no longer necessary, and a relapse was defined as the return of symptoms requiring antibiotics during the late follow-up (LFU) visit. ${ }^{3,20}$ The TOC visit occurred 8-15 days from the last dose of study medication administered, and then the LFU occurred 21-35 days from the last dose of study medication. ${ }^{3,20}$

Table 2. Clinical cure rates in CE patients.

\begin{tabular}{|llll|}
\hline & $\begin{array}{l}\text { CEFTAROLINE } \\
\text { GROUP }\end{array}$ & $\begin{array}{l}\text { CEFTRIAXONE } \\
\text { GROUP }\end{array}$ & $\begin{array}{l}\text { DIFFERENCE, } \\
\text { (95\% CONFIDENCE } \\
\text { INTERVAL [CI]) }\end{array}$ \\
\hline FOCUS 1 & $86.6 \%$ & $78.2 \%$ & $8.4 \%,(1.4-15.4 \%)$ \\
\hline FOCUS 2 & $82.1 \%$ & $77.2 \%$ & $4.9 \%,(-2.5-12.5 \%)$ \\
\hline $\begin{array}{l}\text { Integrated } \\
\text { efficacy } \\
\text { analysis }\end{array}$ & $84.3 \%$ & $77.7 \%$ & $6.7 \%,(1.6-11.8 \%)$ \\
\hline
\end{tabular}


The FOCUS 1 clinical cure rates in CE patients were $86.6 \%$ in the ceftaroline fosamil group versus $78.2 \%$ in the ceftriaxone group (difference, 8.4\%; 95\% confidence interval [CI], 1.4-15.4\%). During FOCUS 2, the clinical cure rates in the $\mathrm{CE}$ population were $82.1 \%$ in the ceftaroline fosamil group versus $77.2 \%$ in the ceftriaxone group (difference, $4.9 \%$; 95\% CI, -2.5-12.5\%). In the integrated efficacy analysis of the two trials, the clinical cure rates in the $\mathrm{CE}$ population were $84.3 \%$ in the ceftaroline fosamil group versus $77.7 \%$ in the ceftriaxone group (difference, 6.7\%; 95\% CI, 1.6-11.8\%). Hence, non-inferiority was established for ceftaroline fosamil when compared to ceftriaxone. Refer to Table 2.3,9,18,19

The FOCUS 1 clinical cure rates in MITTE patients were $83.8 \%$ in the ceftaroline fosamil group versus $77.7 \%$ in the ceftriaxone group (difference, 6.2\%; 95\% confidence interval $[\mathrm{CI}],-0.2-12.6 \%)$. During FOCUS 2, the clinical cure rates in the MITTE population were $81.3 \%$ in the ceftaroline fosamil group versus $75.5 \%$ in the ceftriaxone group (difference, 5.9\%; 95\% CI, $-1.0-12.7 \%)$. The integrated summary reports clinical cure rates in MITTE patients as 82.6 versus $76.6 \%$ for ceftaroline fosamil and ceftriaxone, respectively (difference, 6.0\%; 95\% CI, 1.4-10.7\%), proving ceftaroline fosamil to be non-inferior to therapy with ceftriaxone. Refer to Table 3. 3,9,18,19

Clinical cure rates for the study medication, ceftaroline fosamil, were found to be non-inferior to those of ceftriaxone across all predefined study groups during the entire FOCUS program. Furthermore, clinical relapse rates at the time of LFU were similar between the two study populations for the $\mathrm{CE}$ and MITTE groups as described in the integrated analysis. Therefore, comparable efficacy of ceftaroline fosamil $600 \mathrm{mg}$ administered intravenously every 12 hours was established to ceftriaxone $1 \mathrm{~g}$ administered intravenously every 24 hours in hospitalized patients with CABP requiring IV therapy and having a PORT risk class score of III or IV. ${ }^{3,9,18,19}$

\section{Tolerability and Safety}

Over the course of the FOCUS 1 and FOCUS 2 phase III clinical trials, all enrolled patients (1228 total patients) were followed for treatment-emergent adverse events (TEAEs). Sixhundred and thirteen patients were monitored from the first IV administration of ceftaroline fosamil until the TOC visit, and 615 patients were equally monitored in the ceftriaxone

Table 3. Clinical cure rates in MITTE patients.

\begin{tabular}{|llll|}
\hline & $\begin{array}{l}\text { CEFTAROLINE } \\
\text { GROUP }\end{array}$ & $\begin{array}{l}\text { CEFTRIAXONE } \\
\text { GROUP }\end{array}$ & $\begin{array}{l}\text { DIFFERENCE, } \\
\text { (95\% CONFIDENCE } \\
\text { INTERVAL [CI]) }\end{array}$ \\
\hline FOCUS 1 & $83.8 \%$ & $77.7 \%$ & $6.2 \%,(-0.2-12.6 \%)$ \\
\hline FOCUS 2 & $81.3 \%$ & $75.5 \%$ & $5.9 \%,(-1.0-12.7 \%)$ \\
\hline $\begin{array}{l}\text { Integrated } \\
\text { efficacy } \\
\text { analysis }\end{array}$ & $82.6 \%$ & $76.6 \%$ & $6.0 \%,(1.4-10.7 \%)$ \\
\hline
\end{tabular}

group. Patients who were administered any amount of study medication were included in the safety analysis. Also, serious adverse events (SAEs) including deaths that transpired within 30 days of the last study medication dose received and/or up to the LFU visit were reported. Study medication mean exposure was $6.5 \pm 1.1$ days for both study populations. Patients participated in scheduled laboratory visits from initiation until the TOC visit, while unscheduled laboratory visits were utilized until the LFU visit. $3,18,19,21$

The following safety results are documented from the integrated summary report of the FOCUS 1 and FOCUS 2 clinical studies. The most often reported TEAEs in the ceftaroline fosamil treatment population were diarrhea (4.2\%), headache (3.4\%), and insomnia (3.1\%). Approximately $75 \%$ of patients noticed only mild TEAEs or no TEAEs in either treatment population; therefore, the severity-based distribution of TEAEs was consistent between the two treatment populations. The overall incidence rates of adverse events (AEs) were comparable between the ceftaroline fosamil and ceftriaxone populations, respectively: patients experiencing at least one TEAE (47.0 vs. $45.7 \%$ ), SAE (11.3 vs. $11.7 \%$ ), discontinuation because of an AE (4.4 vs. $4.1 \%)$, or death (2.4 vs. $2.0 \%) .^{3,21}$

Twenty-seven total deaths occurred throughout the FOCUS studies; 15 in the ceftaroline fosamil population and 12 in the ceftriaxone population. Of the deaths recorded, the investigator classified them by type or organ system. Two deaths (one from each population) could have been linked to study medication when appraised by the investigator. Deaths associated with cardiac disorders ( 2 vs. 7 ), infections and infestations ( 3 vs. 1 ), neoplasms ( 4 vs. 0 ), respiratory disorders (4 vs. 3 ), and general disorders such as sudden death and multiple organ disorder (2 vs. 1 ) occurred in the ceftaroline fosamil and ceftriaxone treatment arms, respectively. ${ }^{3,21}$

Seven SAEs were recorded in more than two patients from the ceftaroline fosamil group, which included pneumonia (as defined by worsening or relapse of CABP or nosocomial pneumonia, nine patients), pleural effusion (five patients), pulmonary embolism (five patients), chronic obstructive pulmonary disease (four patients), pyothorax (four patients), respiratory failure (four patients), and malignant lung neoplasm (three patients). The investigator assessed the SAEs, and the majority $(95.7 \%$ in the ceftaroline fosamil population and $91.7 \%$ in the ceftriaxone population) were disregarded as not related to the study medication. Therefore, no safety concerns were expressed. No occurrence of TEAE was documented in more than two subjects in the ceftaroline fosamil population, leading to the discontinuation of the study medication or withdrawal from the clinical trial. However, pneumonia (as defined above), pulmonary embolism, respiratory failure, septic shock, and sudden death each occurred in two patients in the ceftaroline fosamil population, which led to the discontinuation of the study medication or withdrawal from the clinical trial for these reported cases. Ten patients 
in the ceftaroline fosamil population and nine patients from the ceftriaxone population either stopped the study medication or withdrew from the study. The aforementioned SAEs and TEAEs leading to the discontinuation of the study medication revealed an inadequate therapeutic response, which caused prolonged hospitalizations (meets criteria of the SAE definition) or represented AEs that resulted in death (withdrawal from the study). 3,21

In general, ceftaroline fosamil was found to be safe and well tolerated overall. The use of ceftaroline fosamil did not possess any unexpected safety considerations. Ceftaroline fosamil is classified as pregnancy category B. There are no wellcontrolled studies on pregnant women, and it is unknown if ceftaroline is excreted in breast milk. ${ }^{5}$ Therefore, it is recommended by the manufacturer that ceftaroline fosamil only be prescribed if the potential benefit justifies the potential risk to the fetus.

Prescribers should exercise caution in patients with past allergic skin reactions to any other $\beta$-lactam antibiotics and ceftaroline fosamil should be immediately stopped if a reaction develops. Ceftaroline fosamil has a similar safety and tolerability profile as observed with ceftriaxone and other agents of the cephalosporin class. ${ }^{3,5,21}$

\section{Dosage and Administration}

Ceftaroline fosamil is available in 600 and $400 \mathrm{mg}$ reconstitutable vials of powder for injection. ${ }^{5}$ The recommended dose in adults (greater than or equal 18 years of age) with normal renal function ( $\mathrm{CrCl}$ greater than $50 \mathrm{~mL} /$ minute) is $600 \mathrm{mg}$ every 12 hours by IV infusion administered over one hour. Recommended duration of therapy for CABP is $5-7$ days based on the severity of the infection and response to therapy.

Dosing adjustment of ceftaroline fosamil is required in patients with impaired renal function and patients with ESRD on intermittent hemodialysis. ${ }^{5,7,15}$ Patients with mild to moderate renal impairment have an increased AUC of 19 and $52 \%$, respectively, with an increased half-life of 27 and $58 \%$, respectively, after the infusion of a single $600 \mathrm{mg}$ dose. The Cmax was unaltered in patients with mild or moderate renal impairment, but was slightly higher in subjects with severe renal impairment as compared to patients with normal renal function. Therefore, it is recommended that the dosage be adjusted to $400 \mathrm{mg}$ every 12 hours in patients whose $\mathrm{CrCl}$ is greater than 30 but less than or equal to $50 \mathrm{~mL} /$ minute, and further decreased to $300 \mathrm{mg}$ every 12 hours in patients whose $\mathrm{CrCl}$ is greater than or equal to $15 \mathrm{~mL} /$ minute but less than or equal to $30 \mathrm{~mL} /$ minute, and then decreased to $200 \mathrm{mg}$ every 12 hours in patients whose $\mathrm{CrCl}$ is less than $15 \mathrm{~mL} /$ minute with ESRD, including patients receiving hemodialysis. ${ }^{5,8,15}$

Patients receiving intermittent hemodialysis, after receiving a single $400 \mathrm{mg}$ dose of ceftaroline fosamil, had a 167\% higher mean AUC as compared to patients with normal renal function. ${ }^{78}$ Additionally, the Cmax was $74 \%$ higher and the half-life was approximately $123 \%$ longer. Following a four hour hemodialysis session, $21.6 \%(76 \mathrm{mg})$ of ceftaroline was measured in the dialysate. Therefore, ceftaroline fosamil should be administered following hemodialysis on dialysis days. $5,7,15$

\section{Place in Therapy}

The FOCUS trials established that ceftaroline fosamil was non-inferior to ceftriaxone in the treatment of non-ICU patients with CABP; however, it has not been established as preferred use even though the integrated analysis by File et al. found that ceftaroline demonstrated superiority in most of the FOCUS study groups and subgroups. ${ }^{18-20}$ The Average Wholesale Price of one vial of ceftaroline fosamil is $\$ 41$, which corresponds to about $\$ 80$ per day for therapy, and the total cost of treatment is higher as compared to other common treatments such as IV cephalosporins and respiratory fluoroquinolones; additionally, no cost-benefit studies of ceftaroline fosamil in the treatment of CABP have been conducted.

Ceftaroline fosamil does represent a new treatment option for the indication of CABP in the setting of patients at risk for MDROs. Antimicrobial resistance, an aging population, and concomitant comorbidities in the community have changed prescribing practices for CABP. Initial therapy in the inpatient setting may consist of combination therapy and antimicrobial coverage of resistant pathogens. ${ }^{6}$ Utilization of local antibiograms and knowledge of resistance patterns in the community will help to guide selection of antimicrobial agents. Ceftaroline provides antimicrobial activity against wild-type and mutant pathogens responsible for CABP, exhibiting lower MICs for $S$. aureus and $S$. pneumoniae as compared to ceftriaxone and other cephalosporins. Advantages of ceftaroline include its dosing, minimal drug interaction profile, and low resistance to common CABP pathogens, making it an alternative for patients unable to tolerate or respond to other antimicrobial therapies. Patients identified as MRSA colonized or having experienced past episodes of drug-resistant $S$. pneumoniae may benefit from treatment with ceftaroline fosamil initially for $\mathrm{CABP}$, though there are no current clinical data to support its use in this population. ${ }^{3,7}$

\section{Conclusion}

Ceftaroline fosamil represents a new treatment option for patients with CABP, possessing antimicrobial activity against both Gram-positive and Gram-negative bacteria. Antimicrobial treatment for $\mathrm{CABP}$ has changed over the years as a result of antibiotic resistance, an aging population, and patient comorbidities. ${ }^{6}$ Ceftaroline fosamil has been labeled as the first new antibiotic in the IDSA initiative to develop ten new antibiotics by the year 2020. ${ }^{22}$ Practitioners should reserve this antibiotic for patients with risk factors for pathogens with resistance to other antimicrobials that are more cost effective. It does have a spectrum of activity that covers typical pathogens in CABP, but its role in treatment should be reserved for those patients with a high risk of MDROs. 


\section{Author Contributions}

Conceived and designed the experiments: HD, KT. Analyzed the data: HD, KT. Wrote the first draft of the manuscript: HD, KT. Contributed to the writing of the manuscript: HD, KT. Agree with manuscript results and conclusions: HD, KT. Jointly developed the structure and arguments for the paper: HD, KT. Made critical revisions and approved final version: HD, KT. All authors reviewed and approved of the final manuscript.

\section{DISCLOSURES AND ETHICS}

As a requirement of publication the authors have provided signed confirmation of their compliance with ethical and legal obligations including but not limited to compliance with ICMJE authorship and competing interests guidelines, that the article is neither under consideration for publication nor published elsewhere, of their compliance with legal and ethical guidelines concerning human and animal research participants (if applicable), and that permission has been obtained for reproduction of any copyrighted material. This article was subject to blind, independent, expert peer review. The reviewers reported no competing interests. Provenance: the authors were invited to submit this paper.

\section{REFERENCES}

1. Brar NK, Niederman MS. Management of community-acquired pneumonia. Ther Adv Respir Dis. 2011;5(1):61-78.

2. Mandell LA, Wunderink RG, Anzueto A, Bartlett,JG, Campbell GD, Dean NC, et al. Infectious Diseases Society of America/American Thoracic Society consensus guidelines on the management of community-acquired pneumonia in adults. Clin Infect Dis. 2008;44(supp1 2):S27-63.

3. DeBellis HF, Jones MC, Kincaid SE. An update for community-acquired bacterial pneumonia (CABP) pharmacotherapy: what's new and where does it fit? JPharm Pract. 2012;25(6):569-75.

4. Lim L, Sutton E, Brown J. Ceftaroline: a new broad-spectrum cephalosporin. Am J Health Syst Pharm. 2011;68:491-8.

5. Teflaro [Package Insert]. St. Louis, MO: Forest Pharmaceuticals Inc.; 2013.

6. Laudano JB. Ceftaroline fosamil: a new broad-spectrum cephalosporin. J Antimicrob Chemother. 2011;66(3):III11-8.

7. Lodise TP, Law DE. Ceftaroline fosamil in the treatment of community-acquired bacterial pneumonia and acute bacterial skin and skin structure infections. Drugs. 2012;72(11):1473-93.

8. Jorgenson MR, DePestel D, Carver PL. Ceftaroline fosamil: a novel broadspectrum cephalosporin with activity against methicillin-resistant Staphylococcus aureus. Ann Pharmacother. 2011;45:1384-98.
9. Saravolatz LD, Johnson LB. Ceftaroline: a novel cephalosporin with activity against methicillin-resistant Staphylococcus aureus. Clin Infect Dis. 2011;52(9): 1156-63.

10. Kaushik D, Rathi S, Jain A. Ceftaroline: a comprehensive update. Int J Antimicrob Agents. 2011;37:389-95.

11. Jones RN, Farrell DJ, Mendes RE, Sader HS. Comparative ceftaroline activity tested against pathogens associated with community-acquired pneumonia: results from an international surveillance study. J Antimicrob Chemother. 2011;66(3):iii69-80.

12. Critchley IA, Eckburg PB, Jandourek A, Biek D, Friedland HD, Thye DA. Review of ceftaroline fosamil microbiology: integrated FOCUS studies. $J$ Antimicrob Chemother. 2011;66(3):iii45-51.

13. Farrell DJ, Castanheire M, Mendes RE, Sader HS, Jones RN. In vitro activity of ceftaroline against multidrug-resistant Staphylococcus aureus and Streptococcus pneumonia: a review of published studies and the AWARE surveillance program (2008-2010). Clin Infect Dis. 2012;55(3):S206-14.

14. Sader HS, Flemm RK, Jones RN. Antimicrobial activity of ceftaroline-avibactam tested against clinical isolates collected from U.S. medical centers in 2010-2011. Antimicorb Agents Chemother. 2013;57(4):1982-8.

15. Van Wart SA, Forrest A, Kharlton T, Rubino CM, Bhavnani SM, Reynolds DK, et al. Population pharmacokinetics of ceftaroline in patient with acute bacterial skin and skin structure infections or community-acquired bacterial pneumonia. J Clin Pharmacol. 2013;53(11):1155-67.

16. Ge Y, Floren L, Redman R, et al. Single-dose pharmacokinetics (PK) of ceftaroline (PPI-0903) in health subjects. Paper presented at 46th Interscience Conference on Antimicrobial Agent and Chemotherapy; 2006; San Fransisco, CA.

17. Ge Y, Redman R, Floren L, et al. The pharmacokinetics and safety of ceftaroline (PPI-0903) in healthy subjects receiving multiple-dose intravenous (IV) infusions. Paper presented at 46th Interscience Conference on Antimicrobial Agent and Chemotherapy; 2006; San Fransisco, CA.

18. File TM Jr, Low DE, Eckburg PB, Talbot GH, Friedland HD, Lee J, et al. FOCUS 1: a randomized, double-blinded, multicentre phase III trial of the efficacy and safety of ceftaroline fosamil versus ceftriaxone in community-acquired pneumonia. J Antimicrob Chemother. 2011;66 (suppl 3):iii19-32.

19. Low DE, File TM Jr, Eckburg PB, Talbot GH, Friedland HD, Lee J, et al. FOCUS 2: a randomized, double-blinded, multicentre phase III trial of the efficacy and safety of ceftaroline fosamil versus ceftriaxone in community-acquired pneumonia. J Antimicrob Chemother. 2011;66 (suppl 3):iii33-44.

20. File TM Jr, Low DE, Eckburg PB, Talbot GH, Friedland HD, Lee J, et al. Integrated analysis of FOCUS 1 and FOCUS 2: randomized, double-blinded, multicenter phase 3 trials of the efficacy and safety of ceftaroline fosamil versus ceftriaxone in patients with community-acquired pneumonia. Clin Infect Dis. 2010;51:1395-405.

21. Rank DR, Friedland HD, Laudano JB. Integrated safety summary of FOCUS 1 and FOCUS 2 trials: phase III randomized, double-blind studies evaluating ceftaroline fosamil for the treatment of patients with community-acquired pneumonia. J Antimicrob Chemother. 2011;66(suppl 3):iii53-9.

22. Infectious Diseases Society of America. The $10 \mathrm{x}$ ' 20 initiative: pursuing a global commitment to develop 10 new antibacterial drugs by 2020. Clin Infect Dis. 2010;50:1081-3. 